L. Engelmann

\title{
Septisches Lungenversagen
}

in the management of septic pulmonary failure, which extend from fluid restriction, prone positioning to protective ventilation. Protective ventilation consists of low tidal volume, permissive hypercapnia, inspiratory plateau pressure lower than $30 \mathrm{~cm} \mathrm{H}_{2} \mathrm{O}$ and high PEEP. A central problem regarding mechanical ventilation is tailoring the tidal volume to the compliance of the injured lung. A tidal volume of $6 \mathrm{ml} / \mathrm{kg}$ ideal body weight is certainly better than $12 \mathrm{ml} / \mathrm{kg}$; however, this is not the optimum to prevent ventilator-associated lung injury in patients with severe septic pulmonary failure. Extracorporeal lung assist or high frequency oscillation are helpful for effective carbon dioxide elimination if a severe respiratory acidosis ensues as a result of decreasing tidal volume. In case of persistent lifethreatening hypoxia, alveolar recruitment maneuvers and extracorporeal membrane oxygenation can be applied. Further adjuvant measures should be considered in individual cases. Weaning from respirator can be delayed as a result of critical illness polyneuroacute lung injury - ARDS -
Zusammenfassung Sowohl die primäre Infektion der Lunge als auch deren inflammatorische Mitreaktion bei extrapulmonalem Sepsisherd können Ursachen des septischen Lungenversagens sein, das eine über die Jahre unverändert hohe Sterblichkeit aufweist. Dazu kann eine beatmungsinduzierte Lungenschädigung (VALI) das septische Lungenversagen aggravieren. Neben der kausalen Therapie der Sepsis stehen für die Behandlung des septischen Lungenversagens Maßnahmen zur Verfügung, die von der Flüssigkeitsrestriktion über die 4-Seitenlagerung bis hin zur protektiven Beatmung reichen. Protektive Beatmung umfasst niedrige Tidalvolumina, permissive Hyperkapnie, inspiratorische Plateaudrücke unter $30 \mathrm{~cm} \mathrm{H}_{2} \mathrm{O}$ und erhöhten PEEP. Das zentrale Problem der Beatmung, auch beim septischen Lungenversagen, ist das Anpassen des Tidalvolumens an die Compliance der geschädigten Lunge. $6 \mathrm{ml} / \mathrm{kg}$ prädiktives Körpergewicht sind sicher besser als $12 \mathrm{ml} / \mathrm{kg}$, aber nicht das Optimum, um bei schwerem septischen Lungenversagen eine weitere VALI $\mathrm{zu}$ verhindern. Kommt es unter sinkenden Tidalvolumina zur Dekompensation der respiratorischen Azidose stehen mit dem extrakorporalem lung-assist (ECLA) und der Hochfrequenzoszilla\footnotetext{
pathy and/or myopathy.

Key words Sepsis protective ventilation -

adjunctive measures

E-Mail:

Lothar.Engelmann@medizin.uni-leipzig.de

Universitätsklinikum Leipzig A.ö.R.

Intensivmdizin

Liebigstraße 20

04103 Leipzig, Germany

Tel.: $0341 / 9712700$

Fax: $0341 / 9712709$

Die Intensivtherapie bei Sepsis

Herausgegeben von

L. Engelmann (Leipzig)
} 
tionsbeatmung (HFOV) effektive $\mathrm{CO}_{2}$-Eliminationsverfahren zur Verfügung. Der schweren Hypoxie kann mit Rekrutierungsmanövern und extrakorporaler Oxygenierung (ECMO) begegnet werden. Darüberhinaus können im Einzel- fall additive Maßnahmen eingesetzt werden. Das Weaning nach septischem Lungenversagen kann durch eine critical illnessPolyneuropathie/Myopathie überlagert und damit verzögert

sein.
Schlüsselwörter Sepsis Lungenversagen -

Protektive Beatmung Additive Maßnahmen
Das akute Lungenversagen ist der funktionelle Endzustand des auf Schädigung uniform reagierenden Lungenparenchyms. Es unterscheidet zwischen akuter Lungenschädigung (ALI) und ARDS.

Die Häufigkeit von ALI und ARDS liegt zwischen 1,5 und 34 Fällen/100000 Einwohner [1]. Bei einem Drittel der ALI- und ARDS-Patienten ist die Lungenschädigung durch Pneumonie verursacht [2].

Die Inzidenz der schweren Sepsis in der Intensivstation beträgt 150 Fälle/100 000 Patientenjahre, davon entwickeln 30-40\% ein ARDS [1]. Die französische ALIVE-Studie wies eine ICU-Sterblichkeit bei ALI von 22,6 und bei ARDS von $32,7 \%$ auf [3]. Unter den unabhängigen Risikofaktoren für Tod infolge ARDS spielen Sepsis und Leberzirrhose eine zentrale Rolle [4,5]. Die Letalität steht in enger Beziehung zum Multiorganversagen und zur Infektion. Die Sepsis ist die häufigste extrapulmonale Ursache für das Entstehen des akuten Lungenversagens [6]. Während die Sterblichkeit am septischen Lungenversagen in den letzten beiden Dekaden unverändert zwischen 30 und $50 \%$ lag, aber entscheidend von der raschen Sanierbarkeit des septischen Fokus bestimmt wird, ist die Sterblichkeit am Lungenversagen nichtseptischer Ätiologie um 40 auf 29\% gesunken [7], darunter das posttraumatische ARDS sogar auf 10\% [8].

\section{Definition und Diagnostik \\ des akuten Lungenversagens}

Das akute Lungenversagen ist seit der amerikanischeuropäischen Konsensuskonferenz 1992 definiert [9]. Sie differenziert zwischen acute lung injury (ALI) und adult respiratory distress syndrome (ARDS). Die Definition (Tab. 1) ist der kleinste gemeinsame Nenner an diagnostischen Kriterien einer polyätiologischen Schädigung der Lunge und damit hinsichtlich Ätiologie, Therapie und Prognose in hohem Maße unzulänglich. Per definitionem werden mit einem PCWP $\leq 18 \mathrm{mmHg}$ die Lungenstauung infolge Linksherzinsuffizienz und die "fluid lung“ infolge „Überwässerung“ ebenso ausgeschlossen wie chronische Lungenerkrankungen über das Kriterium eines akuten Beginns.

Die Lungenschädigung kann verursacht werden durch eine direkte, primär pulmonale oder eine indirekte, extrapulmonale Lungenparenchymaffektion (Tab. 2; [10]). Beide ätiologisch differenten Formen unterscheiden sich zusätzlich pathophysiologisch und biochemisch, im Röntgenbild und im mechanischen Verhalten der Lunge, woraus sich differente Verhaltensmuster gegenüber therapeutischen Ansätzen ergeben $[11,12]$.

\section{Management \\ des septisch verursachten Lungenversagens}

Die zentrale therapeutische Maßnahme im Managementkonzept des septischen Lungenversagens ist die frühe und konsequente Therapie der der Sepsis zugrundeliegenden Infektion. Im Vordergrund stehen dabei, sofern möglich, die chirurgische oder instrumentelle Herdsanierung. Darüberhinaus beinhaltet das Management eine Bündelung präventiver und therapeutischer Maßnahmen, deren Evidenz- und Empfehlungsgrade in den Leitlinien der Surviving Sepsis Campaign [13] zusammengefasst sind.

Tab. 1 Definition von ALI und ARDS

\begin{tabular}{ll} 
Merkmal & ALI \\
\hline Radiologische Zeichen & Bilaterale Infiltrate in der frontalen Thoraxaufnahme \\
Beginn & Akut \\
Oxygenierung & $\mathrm{paO}_{2} / \mathrm{FiO}_{2}$ (Horovitz-Index) $\leq 300 \mathrm{mmHg}$ \\
& $\begin{array}{l}\text { (unabhängig vom PEEP) } \\
\text { Pulmonaler Kapillardruck (PCWP) }\end{array}$ \\
& $\leq 18 \mathrm{mmHg}$, falls gemessen oder fehlende Zeichen \\
& von Linksherzinsuffizienz
\end{tabular}


Tab. 2 Direkte und indirekte Lungenparenchymaffektionen als Ursache des akuten Lungenversagens

Direkte Lungenparenchymaffektionen

Pulmonale Infektionen (parapneumonisches ARDS)

Beatmung mit inadäquat hohem Tidalvolumina

(ventilatorassoziierte Lungenschädigung, VALI)

Aspiration (Mageninhalt, Salzwasser, Süßwasser)

Inhalation toxischer Gase

Thoraxtrauma

Hohe Sauerstoffpartialdrücke (langdauernd appliziert)

Medikamente mit bevorzugter pulmonaler Verteilung

Aufstieg in große Höhen („Höhenlungenödem“)

Rasche Reexpansion einer längerdauernd komprimierten Lunge
Indirekte Lungenparenchymaffektionen

Sepsis

Schock

Polytrauma

Transfusion-related acute lung injury

Disseminierte intravasale Gerinnung

Lange kardiopulmonale Bypasszeiten

Pankreatitis

Verbrennungen

Allergische Reaktionen

Malaria

Disseminierte Embolien

Narkotika-Intoxikationen

Schädel-Hirn-Trauma
Die Therapie des Lungenversagen umfasst unabhängig von der Ätiologie neben der konsequenten kausalen Therapie des Grundleidens die Schwerpunkte

- Flüssigkeitsrestriktion,

- 4-Seiten-Lagerung,

- protektive Beatmung und

- additive Maßnahmen.

\section{Flüssigkeitsrestriktion}

Der konsequente Flüssigkeitsersatz in der Frühphase der Sepsis gehört zu den Eckpfeilern des Managements von schwerer Sepsis und septischem Schock ([13]; Evidenz Grad B). Eine angemessene Volumensubstitution steht dabei nicht grundsätzlich im Widerspruch mit der Aussage, eine Lunge mit ALI oder ARDS möglichst trocken zu halten. Die Flüssigkeitsrestriktion konkurriert dabei mit dem für eine suffiziente Oxygenierung erforderlichen PEEP, der über eine Vorlastreduktion zum Herzzeitvolumenabfall und zu Kreislaufinstabilität führen kann. Die Anwendung hoher PEEP-Werte erfordert Flüssigkeitsrestriktion auf einem insgesamt höheren Intravasalvolumenbestand. Im Einzelfall kann auch die Zufuhr von Volumen und/oder Katecholaminen erforderlich sein.

Die pathophysiologische Endstrecke des akuten Lungenversagens führt über eine gesteigerte pulmonalkapilläre Permeabilität $\mathrm{zu}$ diffusem alveolärem Ödem und zur Alveolarschädigung [14]. Die transkapilläre Flüssigkeitsfiltration wird neben dem Ausmaß des Kapillarschadens wesentlich vom hydrostatischen Druck bestimmt. Flüssigkeitsrestriktion hat damit das Ziel, die transkapilläre Filtration zu vermindern und die Ödemresorption $\mathrm{zu}$ erleichtern [15]. Obwohl tierexperimentelle Befunde die patho- physiologischen Vorstellungen bestätigen, existieren bislang keine prospektiven klinischen Studien, bei denen ein restriktives Flüssigkeitsmanagement $\mathrm{zu}$ einer Letalitätssenkung geführt hätte. In einer älteren Studie konnte allerdings gezeigt werden, dass bei einer anhand des extravaskulären Lungenwassers im Vergleich zu einer PCWP gesteuerten Patientengruppe die kumulative Flüssigkeitsbilanz niedriger lag, ohne aber Gasaustausch und Letalität $\mathrm{zu}$ verbessern [16]. Dennoch wird in der klinischen Praxis eine negative Flüssigkeitsbilanz bei stabilem Kreislauf angestrebt (Evidenz Grad C; [17]), ohne aber die Splanchnikusperfusion zu gefährden oder ein akutes Nierenversagen $\mathrm{zu}$ induzieren.

\section{Lagerungs-Therapie}

Die pathophysiologische Begründung der Lagerungstherapie, in der Regel die 4-Seiten-Lagerung, ist das Absinken des pulmonalen Ödems in die schwerkraftabhängigen Lungenabschnitte mit entsprechender Störung des regionalen Ventilations-/Perfusions-Verhältnisses, was die meist ohnehin kritische Oxygenierung weiter verschlechtert. Drehen in eine andere Position, besonders aus der Rücken- in die Bauchlage, wirkt diesem Mechanismus passager entgegen.

Die Bauchlagerung führt weitestgehend übereinstimmend in einer Reihe von Studien zur Verbesserung des Gasaustausches [18-21]. In einer prospektiven, randomisierten Studie an 304 Patienten mit ALI und ARDS konnte bislang jedoch kein Nachweis erbracht werden, dass eine 7-stündige Bauchlagerung pro Tag im Vergleich zu Rückenlagerung die Sterblichkeit signifikant senkt [18]. In einer post hoc-Analyse der Subgruppe mit einem Horovitz-In- 
dex $\leq 88 \mathrm{mmHg}$ oder einem SAPS II $>49$ und beiden zusammen zeigte sich ein signifikanter Überlebensvorteil. Bei Zugrundelegung des Endpunktes Überleben ist der Evidenzgrad der Lagerungstherapie mit $\mathrm{C} \mathrm{zu}$ beschreiben. Praktisch ist die Lagerungstherapie eine etablierte therapeutische Maßnahme, mit der in der Regel eine akute, wenn auch passagere Verbesserung der Oxygenierung erreicht wird.

Durch Studien unbeantwortet bleibt die Zeitdauer einer Bauchlagerung. In der eigenen Einrichtung verbleibt der Patient solange in Bauchlagerung wie der Sauerstoffpartialdruck höher als in der vorangegangenen Position ist.

\section{Protektive Beatmung}

Protektiv zu beatmen bedeutet, die Beatmungsparameter so zu wählen, dass eine ventilatorassoziierte Lungenschädigung (VALI) vermieden wird. Mögliche ventilatorassoziierte Lungenschäden sind Baro-, Volu-, Atelekt- und Biotrauma ([22, 23]; Tab. 3). Alle Formen manifestieren sich besonders dann, wenn eine „inhomogene“ Lunge (Nebeneinanderexistieren belüfteter und kollabierter Lungenareale), gemessen an ihrem verbliebenen Lungenvolumen mit zu hohen Tidalvolumina beatmet wird. Das impliziert, dass eine Lunge mit umso niedrigeren Tidalvolumina beatmet werden muss, je kränker sie ist.

Deshalb beinhaltet das Konzept der protektiven

Beatmung

- niedrige Tidalvolumina

- hohen PEEP

- begrenzte inspiratorische Drücke

- umgekehrtes Atemzeitverhältnis

- erhöhte Beatmungsfrequenzen

- permissive Hyperkapnie

- druckgesteuerte Beatmung.

\section{Niedrige Tidalvolumina}

Das zellbiologisch wie tierexperimentell gut belegte Konzept der Anwendung niedriger Tidalvolumina zur Protektion vor VALI konnte bald auch als für die kranke menschliche Lunge effektiv bestätigt werden [24].

Ranieri et al. konnten die Induktion einer die VALI verursachenden pulmonalen inflammatorischen Reaktion überzeugend belegen, indem beatmete Patienten mit ARDS unter einer lungenprotektiven Strategie, verglichen mit ARDS-Patienten unter einer konventionellen Beatmungsstrategie, signifikant niedrigere Entzündungsmarker sowohl in der bronchoalveolären Lavage als auch systemisch aufwiesen [25].

Amato et al. zeigten erstmals in einer Studie den letalitätssenkenden Effekt $(p<0,001)$ einer lungenprotektiven (Vt $<6 \mathrm{ml} / \mathrm{kg}$ Körpergewicht, PEEP oberhalb des an der statischen PV-Kurve bestimmten unteren Inflektionspunktes, Beatmungsspitzendruck maximal $20 \mathrm{~cm} \mathrm{H}_{2} \mathrm{O}$ über PEEP, permissive Hyperkapnie und bevorzugt druckgesteuerter Beatmungsmode) im Vergleich zu einer konventionellen Beatmungsstrategie (bei akzeptabler Oxygenierung möglichst niedrigsten PEEP, Vt $12 \mathrm{ml} / \mathrm{kg}, \mathrm{paCO}_{2}$ zwischen 35 und $38 \mathrm{mmHg}$ [26]).

Seit der Publikation der Ergebnissen des ARDS Network Trials besteht Konsens (Evidenzgrad B) über den Überlebensvorteil (Sterblichkeitsreduktion $9 \%$; [27]) einer low tidal volume-Beatmung (Vt 6,2 $\pm 0,9 \mathrm{ml} / \mathrm{kg}$ berechnetes Körpergewicht; PEEP 9,4 $\pm 3,6 \mathrm{~cm} \mathrm{H}_{2} \mathrm{O}$; Beatmungsfrequenz $29 \pm 7 \mathrm{~min}^{-1}$; $\mathrm{paCO}_{2} \quad 40 \pm 10 \mathrm{mmHg}$ ) verglichen mit einer Beatmung unter Anwendung traditioneller Tidalvolumina (Vt 11,8 $\pm 0,8 \mathrm{ml} / \mathrm{kg} ; \quad$ PEEP $8,6 \pm 3,6 \mathrm{~cm} \mathrm{H}_{2} \mathrm{O}$; Beatmungsfrequenz $16 \pm 6 \mathrm{~min}^{-1} ; \mathrm{paCO}_{2} 35 \pm 8 \mathrm{mmHg}$; [27]). Ausdrücklich sei nochmals darauf verwiesen, dass das NIH-Trial das Tidalvolumen auf $\mathrm{kg}$ errechnetes (ideales) Körpergewicht bezogen hat.

Tab. 3 Ventilatorassoziierte Lungenschädigungen

\begin{tabular}{ll} 
Art der Schädigung & Pathomechanismus und Folgen \\
\hline Barotrauma & $\begin{array}{l}\text { Weniger der Sitzendruck als der hohe transpulmonale Druck (Alveolardruck minus Pleuradruck) } \\
\text { führt zu Druckschädigungen von Alveole und interstitiellem Gewebe } \\
\text { Folge: Pneumothorax, Pneumomediastinum, Hautemphysem, Pneumoperikard, Pneumoretroperitoneum, } \\
\text { interstitielle Lungenödem, Luftembolie }\end{array}$ \\
Starke endinspiratorische Lungendehnung (overdistention) bedingt Alveolarschädigung (ultrastrukturelle Schädigung), \\
Erhöhung der epithelialen und endothelialen Permeabilität und verstärkte Flüssigkeitsfiltration \\
Folge: Lungenödem \\
Schädigung durch rasches Aufeinanderfolgen von Re- und Derekruitment der unteren Atemwege. Die Öffnung erfordert \\
relativ hohe Kräfte und entwickelt Scherstress mit nachfolgender Epithelzerreißung, dies auch bei niedrigen Tidalvolumina \\
Folge: zunehmende Lungenschädigung \\
Lokale inflammatorische Reaktion durch alveolär-interstitielle Schädigung infolge hohe Scherkräfte im Grenzbereich \\
zwischen belüfteten und kollabierten Alveolen \\
Folge: alveolo-interstitielle Entzündung
\end{tabular}

\begin{tabular}{l|l} 
& \\
Intensivmed 5 & 2006
\end{tabular} 
Die Studie vergleicht zwei willkürlich festgelegte Tidalvolumina miteinander. Sie gibt keine Auskunft darüber, ob $\mathrm{Vt}<6 \mathrm{ml} / \mathrm{kg}$ oder an die statische Compliance der jeweiligen Lunge angepasste Tidalvolumina nicht bessere Überlebensraten bringen, wovon unter pathophysiologischem Aspekt auszugehen ist.

Die statische PV-Kurve einer geschädigten Lunge verläuft umso flacher, je geringer die Compliance ist. Gleichzeitig ist in der Regel der untere Inflektionspunkt (s. Abschnitt PEEP) in einen höheren Druckbereich verlagert, so dass der Abschnitt auf der PVKurve, auf dem Volumen ohne Schädigung verabreicht werden kann, ohnehin kleiner wird. Zudem werden in diesem Bereich inadäquat hohe Druckamplituden nur von kleinen Volumenverschiebungen gefolgt. Soll also eine schädigungsfreie Ventilation erfolgen, müssen kleine Tidalvolumina verabreicht werden.

Die Reduktion der Lungenschädigung durch niedrige Tidalvolumina gilt nicht nur für Patienten mit ALI und ARDS, sondern auch für Patienten mit Risikofaktoren für die Entwicklung einer ALI, wozu die Sepsis gehört [28, 29]. Demgegenüber konnten Wrigge und Mitarb. keinen Effekt auf die inflammatorische Reaktion innerhalb von 3 Stunden durch „konventionelle" Tidalvolumina bei elektiven thorakalen und abdominellen Eingriffen nachweisen [30].

Die Anpassung des Tidalvolumens an die verfügbare respiratorische Kapazität ist eines der zentralen Probleme der modernen Intensivmedizin. Klinisch sind die Mittel bescheiden, Überdehnung beatmeter Alveolen zu erkennen.

\section{Hyperinflation und Rekruitment}

Das Erkennen und das Vermeiden von Überdehnung belüftbarer Lungenabschnitte ist deshalb Inhalt gegenwärtiger klinischer Untersuchungen. Am isolierten Ratten-Lungen-Modell [31] und auch am Patienten konnte gezeigt werden, dass das Druckverhalten über der Zeit unter der Bedingung einer Flow-konstanten Inspiration zur Analyse von Rekruitment und Überblähung genutzt werden kann. Die Überlegungen gehen davon aus, dass sich der Quotient $\mathrm{P} / \mathrm{t}$ linear verhält (Stress-Index $=1$ ), wenn sich die Compliance der Lunge mit steigendem inspiratorischen Volumen während konstantem inspiratorischem Flows nicht ändert. Beschreibt die P/t-Beziehung eine nach oben konvexe Kurve (Stress-Index <1), weist das auf eine kontinuierliche alveoläre Rekrutierung und im Falle einer nach unten konvexen Kurve (Stress-Index >1) auf eine alveoläre Überdehnung hin ([32]; Abb. 1).

Ob sich in der klinischen Praxis ein solches Vorgehen als gangbarer Weg erweist, ein der Compliance der Lunge entsprechendes Tidalvolumen zu applizieren und damit eine VALI zu vermeiden, ist derzeit noch nicht vorauszusagen. Nicht zuletzt deshalb, weil in den meisten Einrichtungen gegenwärtig überwiegend druckgesteuerte Beatmungsverfahren eingesetzt werden. Einen solchen Test durchzuführen, würde voraussetzen, die unter druckgesteuerter Beatmung momentan applizierten Beatmungsparameter in einen volumengesteuerten Beatmungsmode $\mathrm{zu}$ übernehmen und die P/t-Beziehung zu analysieren.

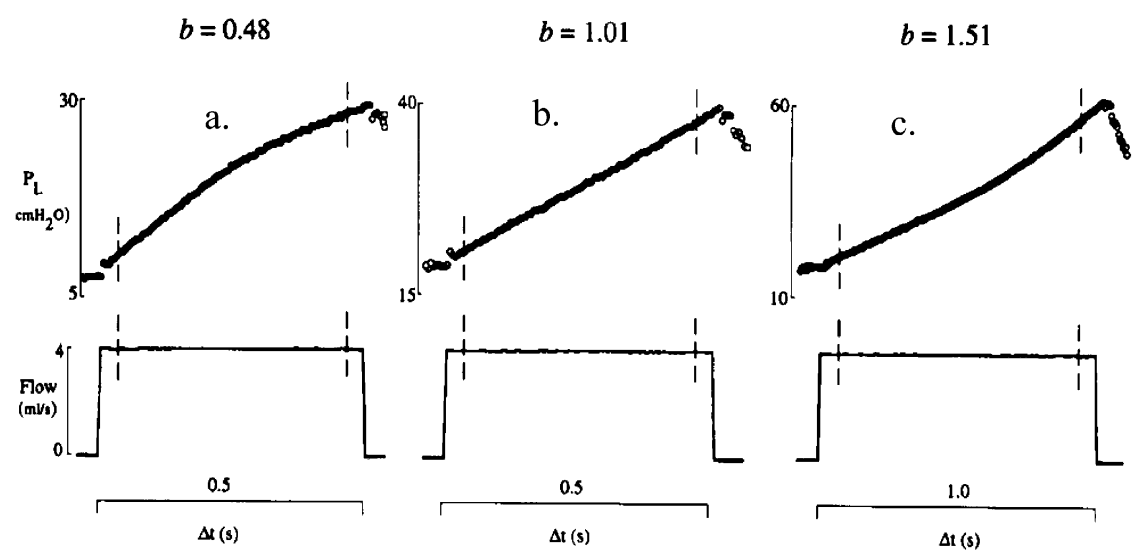

Abb. 1 Dynamische Druck/Zeit (P/t)-Kurve (obere Zeile) bei konstantem inspiratorischem Flow (untere Zeilen): $\mathbf{b}$ zeigt eine gerade P/t-Relation infolge konstanter Compliance bei minimalem Stress; a die nach oben konvexe Kurve weist eine zunehmende Compliance auf und repräsentiert Rekruitment bei geringem Volumenstress: c die nach unten konvexe Kurve repräsentiert eine abnehmende Compliance infolge deutlichen Volumenstresses und bedeutet alveolare Überdehnung. Dabei stellt der Koeffizient b den Stressindex dar, der sich aus der Power-Gleichung $P_{L}=a \cdot t^{b}+b$ ergibt $\left(P_{L}=\right.$ transpulmonaler Druck; $\mathbf{a}=$ Anstieg der $P / t$-Kurve bei $t=1 \mathrm{~s} ; \mathrm{c}=$ Druck bei $\mathrm{t}=0$ ). Aus der Power-Gleichung ergibt sich ein Stress-Index für $\mathbf{a}<1$, für $\mathbf{b}=1$ und für $\mathbf{c}>1$ (nach [31]) 


\section{Hoher PEEP}

Positiv endexspiratorischer Druck (PEEP) ist geeignet, Störungen der Ventilation zu korrigieren, indem das endexspiratorische Kollabieren der Alveolen verhindert wird. Die nachfolgende Inspiration findet offene Alveolen vor, die mit geringem Druckaufwand belüftbar sind. Der zyklische Wechsel von Rekruitment und Derekruitment wird verhindert und damit der Mechanismus für ein Atelekttrauma der Lunge minimiert. Prinzipiell kann der PEEP am Beatmungsgerät eingestellt werden (set PEEP, extrinsischer PEEP) oder durch Wahl geeigneter Beatmungsparameter im kranken Lungenkompartiment erzeugt werden (intrinsischer PEEP). Im ersteren Falle wird die gesamte Lunge, im zweiten Falle nur der kranke Lungenabschnitt unter PEEP gesetzt. Die weiteren Ausführungen beziehen sich zunächst auf den extrinsischen, am Beatmungsgerät einstellbaren PEEP.

\section{Set PEEP}

Die statische PV-Kurve (Abb. 2) weist sowohl im unteren Abschnitt einen Punkt auf (lower inflection point, LIP), bei dessen Überschreiten eine geringe Druckzunahme von stärkerer Volumenzunahme gefolgt wird als unterhalb dieses Druckes. Der Punkt beschreibt den Okklusionsdruck der Atemwege. Der obere Inflektionspunkt (upper inflection point, UIP) stellt den Druck dar, oberhalb dessen die elastischen Eigenschaften des Lungengewebes überfordert sind und die Gesamtlunge überbläht und geschädigt wird.

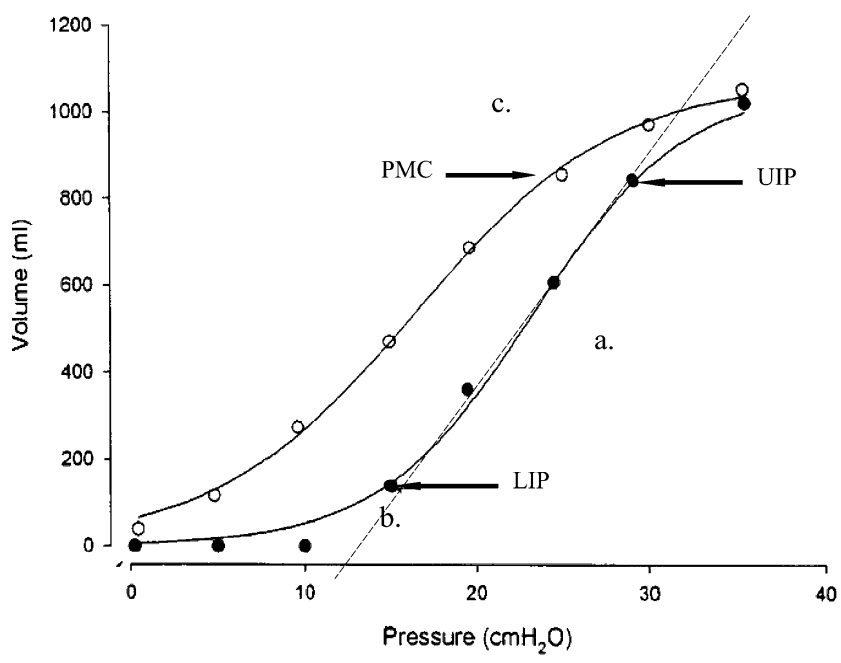

Abb. 2 Vorstellungen zum Rekruitment entlang der statischen Druck-Volumen (PV)-Kurve: a zwischen lower inflection point (LIP) und upper inflection point (UIP); b entlang der Tangente an den Kurvenabschnitt mit konstanter Compliance und $\mathbf{c}$ entlang der Inspiration und Exspiration bis zum point of maximal curvature (PMC)
Die PEEP-Applikation oberhalb des LIP sichert demnach, das Tidalvolumen mit geringeren inspiratorischen Drücken zuzuführen als von einem Druck unterhalb des Inflektionspunktes aus. Physikalisch wird der Vorgang mit dem LaPlaceschen Gesetz beschrieben, wonach der aufzuwendende Druck umso höher sein muss, desto geringer der Radius der Alveole ist. Das fortwährende Kollabieren und Eröffnen der Alveole setzt Schädigungen im Sinne des Atelekttraumas und fördert das Entstehen der VALI.

Die Vorstellungen vom Offenhalten der unteren Atemwege durch einen PEEP oberhalb des LIP sind jedoch Inhalt permanenter Diskussion und offenbar in der Intensivmedizin in der klassischen Form nicht aufrecht zu erhalten. Der LIP repräsentiert den PEEP, oberhalb dessen das Tidalvolumen einer offenen, homogenen Lunge zugeführt werden kann. Bei ALI und ARDS liegt jedoch eine inhomogene Lunge vor, in der Infiltrationen und Ödem schwerkraftabhängig, aber auch -unabhängig, verteilt sind und belüftete wie auch kollabierte Lungenabschnitte nebeneinander bestehen [23, 33]. Der LIP ist auf der PV-Kurve nur bei homogenen, incl. homogen geschädigten Lungen darstellbar. Der LIP zeigt damit lediglich das Ausmaß und die Homogenität der Lungenschädigung, nicht aber den „open-lung“-PEEP an. Die Rekrutierbarkeit der Lunge durch PEEP vollzieht sich offenbar entlang der gesamten PV-Kurve, zumindest entsprechend des bis ZEEP verlängerten Teiles der linearen Compliance. Dabei zeigt der UIP das Ende des Rekruitments und nicht notwendigerweise den Beginn der Überdehnung an [34]. Die Diskussion zum optimalen PEEP bei Lungenschädigung ist weiterhin kontrovers, nicht zuletzt wegen der Koinzidenz von Rekruitment nichtbelüfteter und Hyperinflation belüfteter Lungenabschnitte. So konnte eine kürzliche erschienene Arbeit bei der Wahl des PEEP in der Höhe des exspiratorischen PMC (point of maximal curvature) eine verbesserte Oxygenierung, eine Zunahme der belüfteten und eine Abnahme der nichtbelüfteten Lungenabschnitte sowie eine erhöhte Alveolarstabilität nachweisen. Die Vorteile waren aber verknüpft mit Anstieg des $\mathrm{paCO}_{2}$, des Atemwegsdruckes und des Anteiles überdehnter Lungenabschnitte [35].

Für die klinische Praxis resultiert aus diesen Befunden, einen PEEP oberhalb des LIP zu wählen, um eine ausreichende Oxygenierung $\mathrm{zu}$ gewährleisten. Sofern das Tidalvolumen $6 \mathrm{ml} / \mathrm{kg}$ errechnetes Körpergewichtes beträgt und der endinspiratorische Plateaudruck $30 \mathrm{~cm} \mathrm{H}_{2} \mathrm{O}$ nicht überschreitet, hat die Höhe des PEEP keinen signifikanten Einfluss auf das klinische Ergebnis [36].

Es bestehen unterschiedliche Möglichkeiten den für die jeweilige Lunge besten PEEP zu bestimmen: 
- Bestimmung der Lage der individuellen statischen $P V$-Kurve mittels super-syringe-, rapid airway occlusion oder constant flow-Technik [23, 37] und Applikation eines PEEP oberhalb des unteren Inflektionspunktes [34] - in kritischen Situationen schwer praktikabel, weil Diskonnektion oder unter quasi-statischen Bedingungen zusätzliches Equipment erforderlich sind,

- Aufwärts-Titration des PEEP bei der gewählten $\mathrm{FiO}_{2}$ bis zur Normalisierung des $\mathrm{paO}_{2}$ - klinisch gebräuchlichste PEEP-Wahl,

- Verwendung des NIH-Protokolls [27] - sehr schematisch, relativ niedrige PEEP bei hohen $\mathrm{FiO}_{2}$,

- nach erfolgreichem Rekruitment-Manöver Rücktitration des PEEP bis zum plötzlichen Abfalles des $\mathrm{paO}_{2}$, nochmaliges Rekrutieren und Einstellen des PEEP oberhalb dieses Punktes.

Der PEEP-Aufwärtstitration als dem klinisch üblichen Verfahren sind hämodynamische Grenzen gesetzt. PEEP bedeutet Vorlastreduktion, so dass das Herzzeitvolumen und das Sauerstoffangebot $\left(\mathrm{DO}_{2}\right)$ sinken können, obwohl der $\mathrm{paO}_{2}$ erhöht wurde. Die Suche nach dem „best PEEP“ erfordert damit die Bestimmung des $\mathrm{DO}_{2}$. Klinisch ist man jedoch meist gut beraten, den PEEP zwischen 10 und $15 \mathrm{~cm} \mathrm{H}_{2} \mathrm{O}$ anzusiedeln, wenn dabei eine Kreislaufdepression vermieden wird. PEEP bedeutet aber auch Nachlastreduktion, was besonders im Bereich der kardiologischen Intensivmedizin therapeutisch genutzt werden kann [38, 39].

\section{Intrinsischer PEEP}

Von zunehmendem therapeutischen Interesse ist der intrinsische PEEP $[40,41]$, der im Bereich der kranken alveolo-bronchialen Einheit entsteht und wirkt. Ein solche kranke Einheit ist eine „langsame“ Einheit; die Deflation vollzieht sich wegen der Schädigung verzögert. Damit erfolgt die nachfolgende Inflation auf eine noch nicht abgeschlossene Deflation.

Das verbliebene Volumen entspricht Druck und hält die Alveole offen. Aus pathophysiologischen Vorstellungen heraus wird der intrinsische, vor Ort wirksame PEEP umso höher sein, je höher die Beatmungsfrequenz, je kürzer die Exspirationszeit und je geschädigter die broncho-alveoläre Einheit sind. Damit besteht die Möglichkeit, ein pathophysiologisch begründetes Therapieprinzip nur am Ort der Schädigung $\mathrm{zu}$ applizieren, ohne dass die gesamte Lunge unter PEEP steht. Das gegenwärtig einschränkende Problem der umfänglichen Nutzung von intrinsischem PEEP ist dessen unzulängliches (Messung am Beatmungsgerät in „expiration hold“ bei set-PEEP 0) oder aufwendiges Monitoring (zusätzlich Druckmessung im Ösophagus), um die Gefahr von Überdeh- nung durch air trapping in den einschlägigen Lungenabschnitten zu vermeiden. Gerade bei ARDS-Patienten besteht im Rahmen der protektiven Beatmung durch hohe Beatmungsfrequenzen und verlängerte Inspirationszeiten [40], aber auch durch niedrige Tidalvolumina [42] die Möglichkeit der Induktion von unkontrolliertem intrinsischen PEEP.

Zusammenfassend hat PEEP in die protektive Beatmung Einzug gehalten, weil er (1.) die Ventilation geschädigter Alveolen erleichtert, (2.) das Atelekttrauma verhindert und (3.) die Oxygenierung fördert. Zudem rekrutiert der auf den PEEP aufgesetzte inspiratorische Druck in Abhängigkeit von dessen Höhe.

\section{Begrenzter inspiratorischer Druck}

Die statische PV-Kurve definiert den oberen Inflektionspunkt als den Punkt, bei dessen Überschreitung das elastischen Element der Lunge überdehnt werden kann und vermehrt Barotraumen auftreten. Ein kurzzeitiges Überschreiten wie beim Husten oder im Rahmen eines nur wenige Atemzüge dauernden Rekruitment-Manövers sind offenbar von vernachlässigbarem Einfluss im Vergleich zu längerfristiger Beatmung mit hohen end-inspiratorischen Drücken. In den zur protektiven Beatmung durchgeführten Studien wird ein end-inspiratorischer Plateaudruck von $35 \mathrm{~cm} \mathrm{H}_{2} \mathrm{O}$ in der Regel nicht überschritten. Die Leitlinie der Surviving Sepsis Campaign empfiehlt $30 \mathrm{~cm} \mathrm{H}_{2} \mathrm{O}$ als Plateaudruck und hält zur Vermeidung von VALI die Akzeptanz dieses Druckes für bedeutender als das Tidalvolumen von $6 \mathrm{ml} / \mathrm{kg}$ errechnetes Körpergewicht [13]. Hochgradig compliancegestörte Lungen werden demzufolge eher mit niedrigeren Tidalvolumina als $6 \mathrm{ml} / \mathrm{kg}$ beatmet werden müssen.

\section{Umgekehrtes Atemzeit-Verhältnis}

Die Exspiration dauert normalerweise das 2- bis 3-fache der Inspiration. Die Umkehr des Verhältnisses (inverse ratio ventilation, IRV) über $1: 1$ bis $3: 1$ verkürzt die Exspiration, was die Generierung eines intrinsischen PEEP impliziert. Offenbar liegt in der Induktion eines intrinsischen PEEP die verbesserte Oxygenierung unter IRV begründet [43]. Zugleich erlaubt die verbesserte Oxygenierung die Verringerung des Minutenvolumens, des Spitzendruckes und des PEEP [44]. $\mathrm{Zu}$ beachten sind die Nebeneffekte einer IRV(Reduktion der rechts- und linksventrikulären Vorlast, Anstieg der rechtsventrikulären Nachlast, air trapping in den unter intrinsischen PEEP stehenden Lungenabschnitte). 


\section{Erhöhte Beatmungsfrequenzen}

Die Beatmungsfrequenz sollte dann aus dem Normbereich von 15-20 $\mathrm{min}^{-1}$ erhöht werden, wenn $\mathrm{CO}_{2}$ retiniert wird. Die $\mathrm{CO}_{2}$-Elimination wird von der alveolären Ventilation, entsprechend dem Atemminutenvolumen, bestimmt. Wenn unter protektiver Beatmung das Tidalvolumen der gesunkenen Comliance angepasst reduziert wird, muss die Beatmungsfrequenz erhöht werden, um das Minutenvolumen und damit die $\mathrm{CO}_{2}$-Elimination konstant $\mathrm{zu}$ halten. Im Frequenzbereich zwischen 40 und $60 \mathrm{~min}^{-1} \mathrm{kann}$ das Tidalvolumen dann so gering werden, dass nur noch Totraum ventiliert wird. Praktisch wird das daran erkennbar, dass nach kontinuierlichem Abfall des $\mathrm{paCO}_{2}$ mit steigender Beatmungsfrequenz der $\mathrm{paCO}_{2}$ plötzlich wieder ansteigt. Der Beatmungsfrequenzsteigerung sind damit mit der konventionellen Beatmung Grenzen gesetzt.

Auf die Bedeutung erhöhter Beatmungsfrequenzen für die Induktion eines intrinsischen PEEP, aber auch der damit möglichen Überdehnung langsamer Kompartimente wurde bereits verwiesen.

\section{Permissive Hyperkapnie}

Trotz Atemfrequenzsteigerung wird bei reduziertem Tidalvolumen der $\mathrm{paCO}_{2}$ nicht im Normbereich gehalten werden können. Die konsekutive Hyperkapnie wird dann solange toleriert („erlaubt“, permitted), wie sie metabolisch zumindest teilkompensiert und der $\mathrm{pH}$-Wert über 7,2 [17] gehalten wird (EvidenzGrad C; [13]). Die $\mathrm{paCO}_{2}$-Werte bleiben dabei in der Regel unter $100 \mathrm{mmHg}$. Der Abfall unter den genannten $\mathrm{pH}$-Werte kann zum reduzierten Ansprechen auf Katecholamine führen. Die teilweise propagierte Bikarbonatpufferung der unter einen $\mathrm{pH}$ von $7,2-7,15$ dekompensierten respiratorischen Azidose beinhaltet alle Probleme der Bikarbonatinfusion - das aus dem Bikarbonat entstehende $\mathrm{CO}_{2}$ muss ebenfalls über die Lunge eliminiert werden, die intrazelluläre Azidose wird verstärkt und die meist ohnehin schon hohe Osmolalität der unter Flüssigkeitsrestriktion stehenden Patienten wird durch die Natriumlast weiter erhöht [45]. Offen bleibt zum gegenwärtigen Zeitpunkt, ob die Hyperkapnie oder, wahrscheinlicher, die hyperkapnische Azidose selbst einen protektiven Organeffekt haben $[45,46]$. Die wesentlichen hämodynamischen Effekte der Hyperkapnie bestehen im Anstieg von mittlerem Pulmonalarteriendruck und Herzzeitvolumen, das den Abfall des $\mathrm{paO}_{2}$ infolge Zunahme des intrapulmonalen Shunts kompensiert [46, 47], sofern keine septische Kardiomyopathie vorliegt. Zudem scheinen die hämodynamischen Effekte der Hyperkapnie transient zu sein [46].
Anstelle der Bikarbonat- kann eine THAM-Pufferung vorgenommen werden [48]. Die Progredienz der respiratorischen Azidose erfordert dann aber den Einsatz von Systemen zur $\mathrm{CO}_{2}$-Eliminiation.

\section{Druckgesteuerte Beatmung}

Druckgesteuerte (PC) Beatmung besitzt gegenüber volumengesteuerter (VC) theoretisch mehrere Vorteile. (1.) Das PC- applizierte Tidalvolumen ist abhängig von der Compliance der individuellen Lunge und beinhaltet die geringere Überblähungsgefahr als VCBeatmung. Damit sollte die Gefahr eines Biotraumas per se geringer sein. (2.) Im Falle von Rekrutierung während des inspiratorischen Druckanstieges wird bei PC-Beatmung bis zum Beginn der Exspiration über einen dezelerierenden Flow Frischluft zugeführt, während bei VC-Beatmung mit konstantem Flow nach Applikation des vorgegebenen Tidalvolumens im Falle von Rekruitment nur Luft aus ventilierten in rekrutierte Alveolen gelangen kann. „Pendelluft" hat einen geringeren $\mathrm{O}_{2^{-}}$und einen höheren $\mathrm{CO}_{2}$-Gehalt als Frischluft.

Bislang gibt es nur wenige Belege für das Postulat einer vorteilhafteren PC-Beatmung [49] in klinischen Studien [50, 51]. In den ARDS-Studien mit Nachweis einer Letalitätssenkung durch protektive Beatmung wurde von Amato et al. [26] eine PC-Beatmung bevorzugt, während im ARDS-Network-Trial [27] VCBeatmung angewendet wurde.

\section{Maßnahmen \\ jenseits der Grenzen protektiver Beatmung}

Hier sollen Möglichkeiten und Algorithmen aufgezeigt werden, die nach derzeitigem Wissenstand dann einsetzbar sind, wenn die protektive Beatmung in der vorstehenden Form die Grenzen des mit dem Leben vereinbarbaren Gasaustausches erreicht hat. Die Evidenzgrade sind entsprechend niedrig. Im Einzelnen sind zu nennen:

- extracorporal lung assist (ECLA; Hersteller: Novalung $\mathrm{GmbH}$, Hechingen, Deutschland)

- Hochfrequenzoszillations-Ventilation (HFOV)

- Rekruitment-Manöver

- ECMO

\section{Extracorporaler „lung assist"}

Mit diesem Verfahren gelingt eine effektive $\mathrm{CO}_{2}$-Elimination über eine extrakorporale Kapsel, durch deren Kapillaren sich der arterio-venöse Blutfluss pum- 


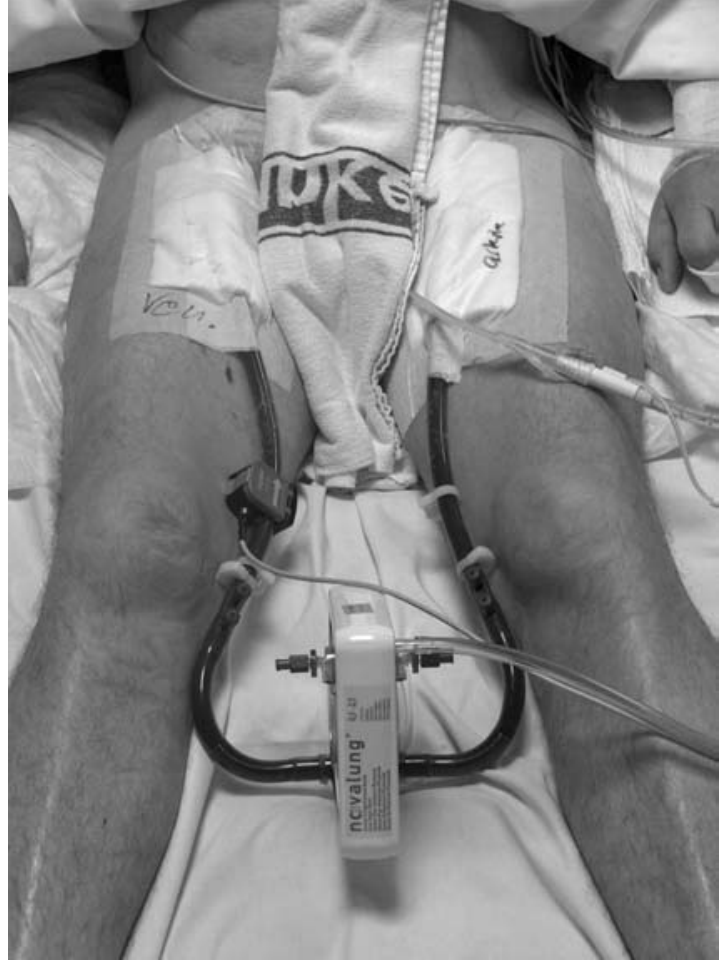

Abb. 3 ECLA (extracorporeal lung assist) im Einsatz: Das pumpenlose System führt das arterielle Blut über Kapillaren in der Kapsel, die von Sauerstoff oder Druckluft umströmt werden und über die sich die $\mathrm{CO}_{2}$-Elimination vollzieht, zurück in den venösen Schenkel

penlos vollzieht (Abb. 3). Um die Kapillaren wird ein Gasfluss (Druckluft oder besser Sauerstoff aus der Wandarmatur) zwischen 12 und $18 \mathrm{l} / \mathrm{min}$ geführt, der das $\mathrm{CO}_{2}$ auswäscht. Der Grad der $\mathrm{CO}_{2}$-Elimination hängt zudem vom Blutfluss über die Kapillaren ab. Die Oxygenierung wird damit nur marginal beeinflusst. Die Oxygenierung vollzieht sich über Beatmungsgerät und Lunge und hängt von der $\mathrm{FiO}_{2}$ und in besonderem Maße vom PEEP ab. Mit der Anlage der Kapsel kann die Lunge mit niedrigsten Tidalvolumina $(<1 \mathrm{ml} / \mathrm{kg}$ Körpergewicht $)$, beinahe apnoisch, ventiliert werden. Tidalvolumen, PEEP und Beatmungsfrequenz können so gewählt werden, dass die derzeitigen Kriterien einer protektiven Beatmung in idealer Weise erfüllt werden. Die Indikationen für die Anwendung des extrakoporalen lung-assist bestehen demnach in der protektiven Beatmung bei schwerst compliancegestörter Lunge und in der $\mathrm{CO}_{2}$-Elimination bei dekompensierter permissiver Hyperkapnie. Die klinischen Erfahrungen beziehen sich auf Einzelfälle und kleine Beobachtungsstudien [52].

\section{Hochfrequenzoszillations-Ventilation (HFOV)}

Während im Rahmen protektiver Beatmung die Beatmungsfrequenzen bis kurz unterhalb der Totraumventilation (40-60 $\mathrm{min}^{-1}$ ) geführt werden, liegen die Frequenzen bei der HFOV im Bereich 300-2400 Zyklen/min [53]. Die Frequenz und der Hub des Oszillators bestimmen die $\mathrm{CO}_{2}$-Elimination, die Höhe des einstellbaren Atemwegsmitteldruckes und die $\mathrm{FiO}_{2}$ die Oxygenierung. Der Oszillator ersetzt das konventionelle Beatmungsgerät. Die physikalischen Vorgänge, über die sich der Gasaustausch vollzieht, sind letztendlich noch nicht restlos geklärt [54]. Mit der HFOV werden Quasi-Tidalvolumina generiert, die in der Lungenperipherie einer nahezu apnoischen Ventilation entsprechen.

In der bislang einzigen randomisierten Studie an 75 Patienten unter HFOV und 73 Patienten unter konventioneller Beatmung konnte eine nichtsignifikant höhere Überlebenrate unter HFOV gezeigt werden [55]. Die Ergebnisse sind dahingehend zu interpretieren, dass offenbar mit der HFOV eine weitere Therapieoption jenseits konventioneller protektiver Beatmung besteht, die Lunge mit extrem kleinen Tidalvolumina $\mathrm{zu}$ beatmen und effektiv $\mathrm{CO}_{2} \mathrm{zu}$ eliminieren.

\section{Rekruitment-Manöver}

Rekruitment-Manöver dienen dem Ziel, bei kritischer Hypoxie atelektatische Alveolen wiederzubelüften. Verschiedene Techniken wurden untersucht: Seufzer [56], airway pressure release-Ventilation [57], volume recruitment-Manöver [58] und das „Lachmann-Manöver" (Abb. 4; [59]).

Letzteres hat in der deutschen Intensivmedizin Verbreitung gefunden $[60,61]$. Die modifizierten „Lachmann-Manöver" sind anderenorts beschrieben [62].

Es geht von der pathophysiologischen Vorstellung aus, dass zur Eröffnung atelektatischer Alveolen zunächst hohe Eröffnungsdrücke notwendig sind, um dann unter Druckrücknahme in den Normbereich die eröffneten Alveolen mit entsprechend hohem PEEP offen zu halten. Den Hintergrund bildet auch hier das LaPlacesche Gesetz. Sicherlich hängt die Höhe des erforderlichen Eröffnungsdruckes von der Genese der Atelektase ab. Bei schwerem parapneumonischen ARDS sind gelegentlich Spitzendrücke bis $80 \mathrm{~cm} \mathrm{H} \mathrm{H}_{2} \mathrm{O}$ erforderlich; die Atelektasen beim Polytrauma sind mit niedrigeren Drücken zu eröffnen [60]. Schließlich hängt die Erfolgsrate des Lachmann-Manövers auch von der ARDS-Dauer ab $(1,54 \pm 0,97$ Tage bei erfolgreichem vs. $6,00 \pm 5,58 \mathrm{Ta}-$ ge bei erfolglosem Manöver, $\mathrm{p}=0,002$; eigene, unveröffentlichte Befunde). 
Abb. 4 Rekruitment-Manöver nach Lachmann. Inspiratorische Spitzendrücke von $70 \mathrm{~cm} \mathrm{H}_{2} \mathrm{O}$ rekrutieren partiell bis zum $\mathrm{paO}_{2}$ von $240 \mathrm{mmHg}$ Der Effekt lässt sich mit einem PEEP von $25 \mathrm{~cm} \mathrm{H}_{2} \mathrm{O}$ stabilisieren. Danach erfolgt die Reduktion der $\mathrm{FiO}_{2}$ bis zu einem normalen $\mathrm{paO}_{2}$ (PARATREND-online-Registrierung)

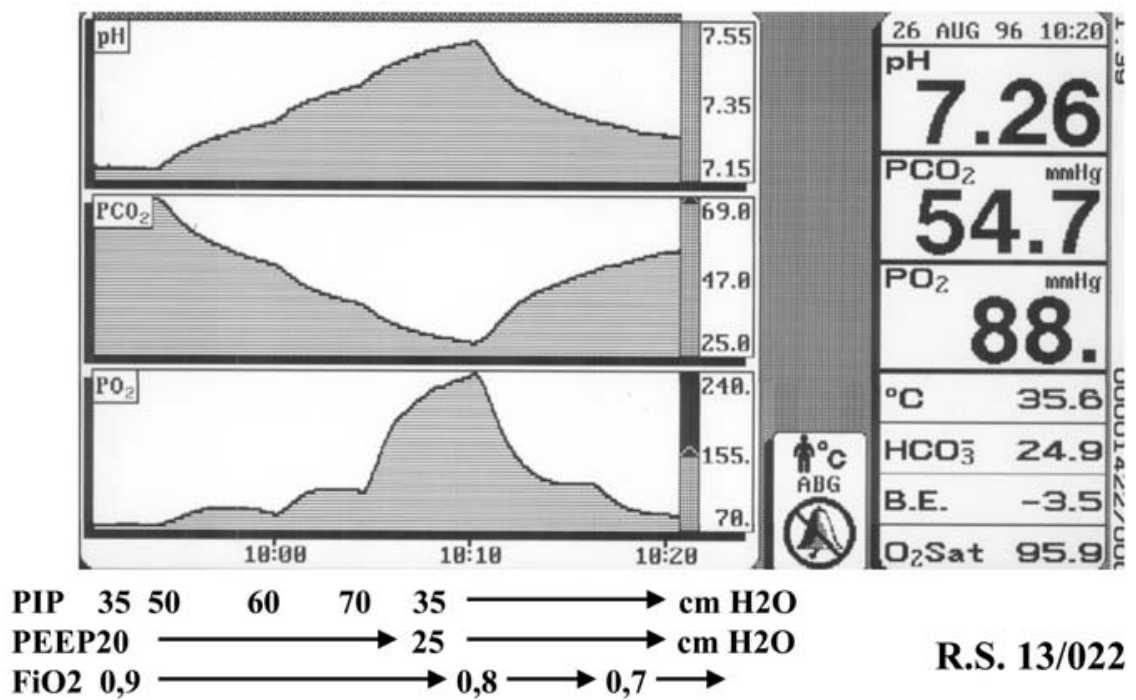

Die wichtigsten Argumente gegen die Anwendung des "Lachmann-Manövers" sind auf die Applikation der Spitzendrücke gerichtet, die einer protektiven Beatmung zu widersprechen scheinen.

Die Induktion eines Biotraumas kann derzeit nicht ausgeschlossen werden, weshalb zum jetzigen Zeitpunkt grundsätzlich gilt, das „Lachmann-Manöver" nur dann durchzuführen, wenn die Optionen protektiver Beatmung versagen und die kritisch schlechte Oxygenierung das zentrale Problem darstellt.

Wichtigstes Argument gegen die Induktion eines Biotraumas ist die Kürze des Rekruitmentmanövers. In der Regel genügen maximal 10 Beatmungszyklen für ein suffizientes Manöver. Bislang vorliegende Daten zur Induktion einer inflammatorischen Reaktion durch „nichtprotektive“ Beatmung konnten den Anstieg proinflammatorischer Zytokine im Plasma und den Abfall derselben in der bronchoalveolären Lavage bei "protektiv“ beatmeten Patienten nach 24-40 Stunden belegen [25]. Sowohl lungengesunde, als auch Patienten, die im Rahmen chirurgischer Eingriffe mit hohen Tidalvolumina beatmet wurden, zeigen nach 1 Stunde bzw. 3 Stunden keine signifikanten Änderungen der Plasmazytokinspiegel [63, 30]. Bei Kleinkindern führte eine 2-stündige Narkosebeatmung mit einem Tidalvolumen von $10 \mathrm{ml} / \mathrm{kg}$ KG zur Triggerung einer inflammatorischen Reaktion in der Lunge [64]. In den tierexperimentellen Untersuchungen Tremblays [65] wurden Rattenlungen 2 Stunden differenten Beatmungsstrategien ausgesetzt, um die proinflammatorische Reaktion zu erfassen. Eine NF- $\kappa$ B-Induktion in isolierten perfundierten Mäuselungen wird nach 60 min nichtprotektiver Beatmung beobachtet [66].
In der Sequenz protektive Beatmung, permissive Hyperkapnie, ECLA ist ein "open-lung"-Manöver möglicherweise dann indiziert, wenn die Oxygenierung zum kritischen Moment in der Beatmung wird. Studien hierzu liegen nicht vor.

\section{ECMO}

Extrakorporale Membranoxygenierung verbindet protektive Beatmung mit $\mathrm{CO}_{2}$-Elimination und Oxygenierung. Der apparative Aufwand ist um ungleiches höher als bei der ECLA, die nicht über die Möglichkeit der direkten Oxygenierung verfügt. Aufgrund des technischen Equipments, der damit verbundenen Komplikationsmöglichkeiten, aber auch der Erfahrungen wegen, wird ECMO von wenigen Zentren vorgehalten. Wie bei der Polyätiologie des Lungenversagens $\mathrm{zu}$ erwarten, hat ECMO in randomisierten, prospektiven Studien nicht zur signifikanten Senkung der Sterblichkeit am ARDS geführt [67, 68]. Im individuellen Falle kann ECMO das ultimative Therapieprinzip sein. Von den ECMO-Zentren werden „fast-“ und „slow-entry“-Kriterien formuliert [69], die sich zentrumsabhängig unterscheiden.

\section{Additive Maßnahmen}

Unter die additiven Maßnahmen werden hier Surfactant-Applikation, Prostaglandin-Infusion und NOInhalation subsumiert. 


\section{Surfactant-Applikation}

Die hohen Erwartungen aus den tierexperimentellen Untersuchungen und dem Einsatz bei Neonaten an den Ersatz des bei Lungenschädigung quantitativ verminderten, durch Proteine inhibierten und funktionell veränderten Surfactant haben sich bei Patienten mit ARDS bislang in Bezug auf eine verbesserte Überlebensrate in keiner randomisierten, placebokontrollierten Studie bestätigt [70-72], obwohl sich der Gasaustausch erheblich verbessert [73]. Gegenwärtig besteht keine Indikation für eine lungensystemische Applikation von Surfactant. Dagegen kann seine dosisadaptierte Instillation in atelektatische Lungenabschnitte $\mathrm{zu}$ einer Verbesserung der Oxygenierung führen. Hierzu liegen Einzelberichte vor $[74,75]$.

\section{Prostaglandin-Infusion}

Prostaglandin $\mathrm{E}_{1}$ ist ein pulmonaler und systemischer Vasodilatator, der über eine Down-Regulation der Neutrophilen-vermittelten Immunantwort direkt in das Inflammationsgeschehen bei ARDS eingreift. Die Prostaglandin-Infusion hat den negativen $\mathrm{Ne}$ beneffekt der systemischen Vasodilatation, der beim sepsisinduzierten Lungenversagen eher unerwünscht ist. In randomisierten, placebo-kontrollierten Studien mit liposomalem Prostaglandin $\mathrm{E}_{1}$ konnten zwar Oxygenierung, und Lungencompliance verbessert und die Ventilatorabhängigkeit gesenkt werden [76], aber ein Überlebensvorteil wurde nicht erreicht [77].

Während die systemisch verabreichten Prostaglandine $\mathrm{PGE}_{1}$ und $\mathrm{PGI}_{2}$ sowie das länger wirkende Iloprost wegen der genannten systemischen Dilatation ihre Indikation eher bei der schweren pulmonalen Hypertonie besitzen, besteht für aerosoliertes $\mathrm{PGI}_{2}$ eine pulmonale und intrapulmonale Selektivität [78], deren Bedeutung für die Therapie des ARDS in Studien überprüft werden muss.

\section{NO-Inhalation}

Inhaliertes Stickstoffmonoxyd ist ein selektiver pulmonaler Dilatator im Bereich ventilierter Alveolen mit dem Effekt der Besserung eines gestörten Ventilation-/Perfusionsverhältnisses, der Hypoxämie und der pulmonalen Hypertension. Das Wirkungsprofil setzt Erwartungen in die Behandlung des ARDS. Verbesserungen der Oxygenierung lassen sich bei Dosierungen von 5-10 ppm häufig nachweisen, was in kritischen Oxgenierungssituationen durchaus die Funktion des rettenden Strohhalmes haben kann (Abb. 5).

Bislang konnte aber kein signifikanter letalitätssenkender Effekt nachgewiesen werden [79, 80], was aber bei NO verwendenden Intensivstationen nicht $\mathrm{zu}$ dessen Elimination aus dem therapeutischen Repertoir geführt hat. Ein europäisches Expertengremium hält die inhalative NO-Applikation im Rahmen
Abb. 5 Effekt von 10 ppm Stickstoffmonoxyd auf die Oxygenierung bei einem Patienten mit ARDS in der online-Restrierung mittels PARATREND (Hersteller: Biomedical Sensors Ltd, High Wycombe, England)

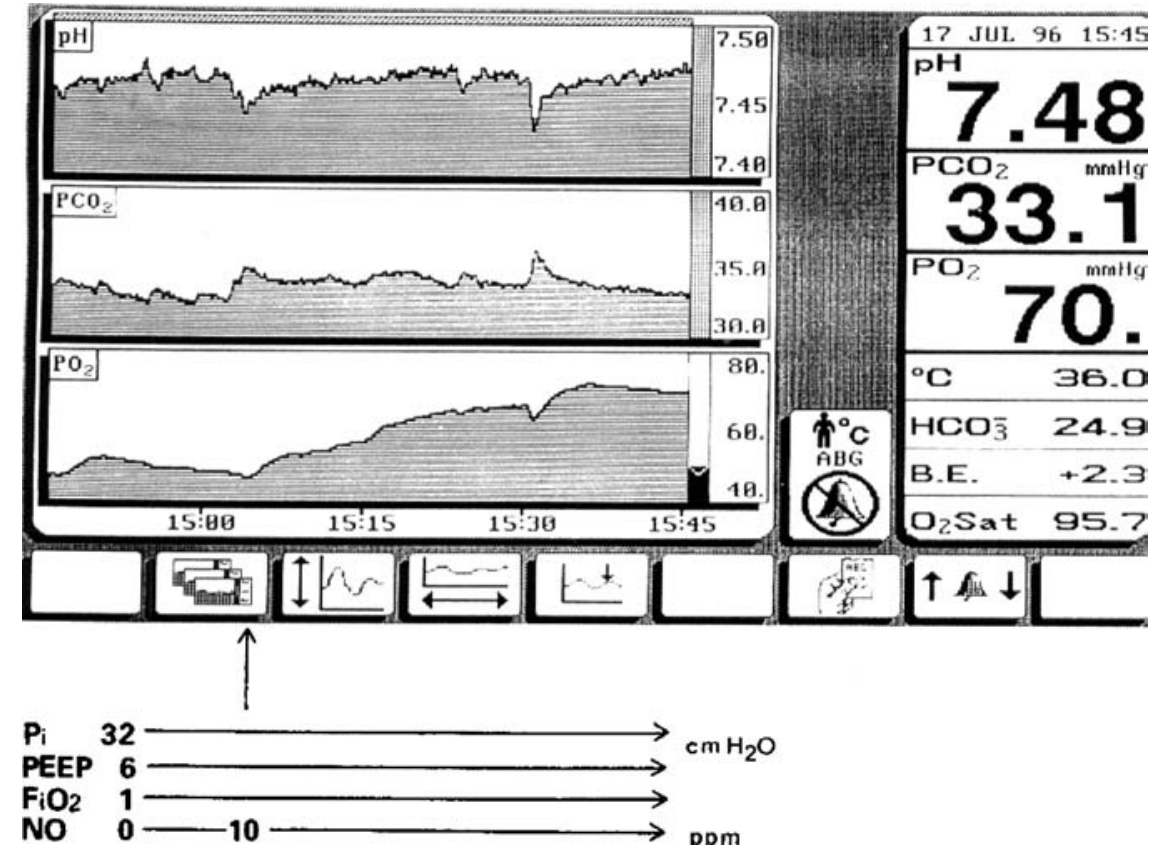


der Beatmung bei ARDS für nicht indiziert, auch nicht als Rescue-Maßnahme bei therapierefraktärer Hypoxämie [81].

\section{Beatmungsentwöhnung}

Für die Beatmungsentwöhnung nach septischem Lungenversagen gelten zunächst die gleichen Grundregeln der Entwöhnung wie für jedes Weaning bei anderer Indikation auch. Als Leitsätze gelten: Beatmungsentwöhnung beginnt mit der Intubation, zum frühesten Zeitpunkt die Beatmung mit einem assistierten Mode führen, die Analgosedierung nach dem Tagesziel gestalten, die Grundsätze der protektiven Beatmung auch in der Phase des Weanings beachten, keine Hypoxie zulassen und zum frühest möglichen Zeitpunkt extubieren. Der Zeitpunkt der Extubation wird durch die Möglichkeit der Fortsetzung der Beatmung über BiPAP-Maske modifiziert. Die Verfechter eines programmierten Weanings werden täglich die Spontanatmungstests einfügen, die auf Ely et al. zurückgehen [82]. Hier wurden die zu entwöhnenden Patienten einem täglichen Spontanatmungstest unterzogen, wenn folgende Bedingungen erfüllt waren:

- $\mathrm{paO}_{2} / \mathrm{FiO}_{2}>200 \mathrm{mmHg}$,

- PEEP $\leq 5 \mathrm{~cm} \mathrm{H}_{2} \mathrm{O}$,

- adäquater Hustenstoß bei Absaugung,

- shallow-breathing-index (Atemfrequenz dividiert durch Tidalvolumen in Litern) $\leq 105$ Atemzüge/ $\mathrm{min} / \mathrm{l}$ und

- keine Vasopressoren.

Waren diese Bedingungen erfüllt, erfolgte entweder über ein T-Stück oder bei CPAP $5 \mathrm{~cm} \mathrm{H}_{2} \mathrm{O}$ ein Spon-
Tab. 4 Abbruchkriterien des täglichen Spontanatmungsscreenings

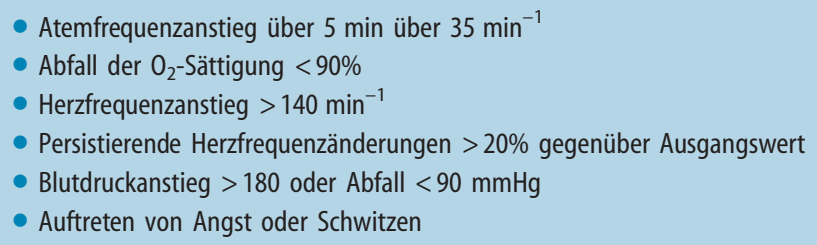

tanatmungsversuch, der beim Auftreten von Abbruchkriterien (Tab. 4) wieder durch Beatmung ersetzt wurde, um am nächsten Tag erneut zu screenen. Der Test wurde bei einer Spontanatmung über 2 Stunden als erfolgreich bewertet. Das Screening wird in einem Zeitfenster verminderter oder unterbrochener Analgosedierung durchgeführt, was impliziert, dass zum Zeitpunkt des geplanten Weanings die Analgosedierung mit Medikamenten erfolgt, die über eine kurze Halbwertzeit verfügen.

Solchermaßen verfahren, konnte die Weaningdauer im Mittel von 3 auf einen Tag reduziert werden $(\mathrm{p}<0,001)$, die Dauer der Beatmung sank von 6 auf 4,5 Tage $(\mathrm{p}<0,003)$ und die Häufigkeit der Reintubationen reduzierte sich [83]. Die verkürzte Intubationsdauer ist besonders bei Sepsis unter dem Aspekt einer attributiven Sterblichkeit infolge tubusassoziierter Lungenschädigung von erheblicher Bedeutung.

Die skizzierten Maßnahmen sind im Detail in den Guidelines von ACCP, AARC und ACCM dargestellt und kommentiert [84].

Die Sepsis kann den Weaningprozess prolongieren, wenn eine critical illness-Polyneuro-/Myopathie den Verlauf kompliziert.

\section{Literatur}

1. Goss $\mathrm{CH}$, Brower RG, Hudson LD et al (2003) Incidence of acute lung injury in the United States. Crit Care Med 31:1607-1611

2. Schwarz MI, Albert RK (2004) "Imitators" of the ARDS. Implications for diagnosis and treatment. Chest 125: $1530-1535$

3. Brun-Buisson C, Minelli C, Bertolini $\mathrm{G}$ et al (2004) Epidemiology and outcome of acute lung injury in European intensive care units. Results from ALIVE study. Intensive Care Med 30:51-61
4. Monchi M, Bellenfant F, Cariou A et al (1998) Early predictive factors of survival in the acute respiratory distress syndrome. Am J Respir Crit Care Med 158:1076-1081

5. Tentlow Chest 2001

6. Milberg JA, Davis DR, Steinberg KP, Hudson LD (1995) Improved survival of patients with acute respiratory distress syndrome (ARDS) 1983-1993. JAMA 273:306-309

7. Stapleton RD, Wang BM, Hudson LD et al (2005) Causes and timing of death in patients with ARDS. Chest 128:523-553

8. Vincent ICM 2003
9. Bernard GR, Artigas A, Brigham KL et al (1994) The American-European Consensus Conference on ARDS. Definitions, mechanisms, relevant outcome, and clinical trail coordination. Am J Respir Crit Care Med 149:818824

10. Gattinoni L, Pelosi P, Suter $\mathrm{P}$ et al (1998) Acute respiratory distress syndrome caused by pulmonary and extrapulmonary disease. Different syndromes? Am J Rspir Crit Care Med 158:3-11

11. Pelosi P, D'Ònofrio D, Chiumello D et al (2003) Pulmonary and extrapulmonary acute respiratory distress syndrome are different. Eur Respir J 22(Suppl 42):48s-56s 
12. Albaiceta GM, Taboada F (2005) Pressure-volume loops in ARDS: further evidence of differences between pulmonary and extrapulmonary origin. Intensive Care 12:73-80

13. Dellinger RP, Carlet JM, Masur $\mathrm{H}$ et al (2004) Surviving Sepsis Campaign guidelines for management of severe sepsis and septic shock. Crit Care Med 32:858-873

14. Schuster DP (1995) What is the acute lung injury? What is ARDS? Chest 107:1721-1726

15. Benzing A, Mols G, Geiger K (2001) Flüssigkeitstherapie bei akutem Lungenversagen. Intensivmed 38:690-698

16. Mitchell JP, Schuller D, Calandrino FS, Schuster DP (1992) Improved outcome based on fluid management in critically ill patients requiring pulmonary artery catheterization. Am Rev Respir Dis 145:990-998

17. Sevransky JE, Levy MM, Marini JJ (2004) Mechanical ventilation in sepsis-induced acute lung injury/acute respiratory distress syndrome: an evidence-based review. Crit Care Med 32(Suppl):S548-S553

18. Gattinoni L, Tognoni G, Pesenti A et al (2001) Effect of prone positioning on the survival of patients with acute respiratory failure. N Engl J Med 345: 568-573

19. Beuret P, Carton MJ, Nourdine K et al (2002) Prone position as prevention of lung injury in comatose patients: a prospective, randomized, controlled study. Intensive Care Med 28:564-569

20. McAuley DF, Gies S, Fichter $\mathrm{H}$ et al (2002) What is the optimal duration of ventilation in the prone position in acute lung injury and acute respiratory distress syndrome? Intensive Care Med 28:414-418

21. Gainnier M, Michelet P, Thirion X et al (2003) Prone positioning and positive end-expiratory pressure in acute respiratory distress syndrome. Crit Care Med 31:2719-2726

22. Slutsky AS (1999) Lung injury caused by mechanical ventilation. Chest 116 : 9S-15S

23. Terragni PP, Rosboch GL, Lisi A et al (2003) How respiratory system mechanics may help in minimising ventilator-induced lung injury in ARDS patients. Eur Respir J 22(Suppl 42): 15S-21S

24. Hickling KG, Walsh J, Henderson S, Jackson R (1994) Low mortality rate in adult respiratory distress syndrome using low-volume, pressurelimited ventilation with permissive hypercapnia: a prospective study. Crit Care Med 22:1568-1578
25. Ranieri VM, Suter PM, Tortorella C et al (1999) Effect of mechanical ventilation on inflammatory mediators in patients with acute respiratory distress syndrome. A randomized controlled trial. JAMA 282:54-61

26. Amato MBP, Barbas CSV, Medeiros DM et al (1998) Effect of a protective-ventilation strategy on mortality in the acute respiratory distress syndrome. N Engl J Med 338:347-354

27. ARDS Network Trial (2000) Ventilation with lower tidal volume as compared with traditional volumes for acute lung injury and the acute respiratory distress syndrome. $\mathrm{N}$ Engl J Med 342:1301-1308

28. Gajic O, Dara SI, Mendez JL et al (2004) Ventilator-associated lung injury in patients without acute lung injury at the onset of mechanical ventilation. Crit Care Med 32:18171824

29. Gajic O, Frutos-Vivar F, Esteban A et al (2005) Ventilator settings as a risk factor for acute respiratory distress syndrome in mechanivally ventilated patients. Intensive Care Med 31:922926

30. Wrigge $\mathrm{H}$, Uhlig $\mathrm{U}$, Zinserling $\mathrm{J}$ et al (2004) The effect of different ventilatory settings on pulmonary and systemic inflammatory responses during major surgery. Anesth Analg 98:775781

31. Ranieri VM, Zhang H, Mascia L et al (2000) Pressure-time curve predicts minimally injurious ventilatory strategy in an isolated rat lung model. Anesthesiology 93:1320-1328

32. Grasso S, Terragni P, Mascia L et al (2004) Airway pressure-time curve profile (stress index) detects tidal recruitment/hyperinflation in experimental acute lung injury. Crit Care Med 32:1018-1027

33. Rouby JJ, Lu Q, Vieira S (2003) Pressure/volume curves and lung computed tomography in acute respiratory distress syndrome. Eur Respr J 22(Suppl 42):27S-36S

34. Maggiore SM, Richard JC, Brochard L (2003) What das been learnt from $\mathrm{P} / \mathrm{V}$ curves in patients with acute lung injury/acute respiratory distress syndrome. Eur Respir J 22(Suppl 42):22S-26S

35. Albaiceta GM, Luyando LH, Parra D et al (2005) Inspiratory vs expiratory pressure-volume curves to set end-expiratory pressure in acute lung injury. Intensive Care Med 31:13701378
36. The National Heart, Lung, and Blood Institute ARDS Clinical Trials Network (2004) Higher versus lower positive end-expiratory pressures in patients with the acute respiratory distress syndrome. N Engl J Med 351: 327-336

37. Lu Q, Rouby JJ (2000) Measurement of pressure-volume curves in patients on mechanical ventilation. Minerva Anestesiol 66:367-375

38. Smiseth OA, Thomoson $\mathrm{CR}$, Ling $\mathrm{H}$ et al (1996) A potential clinical method for calculating transmural left ventricular filling pressure during positive end-expiratory pressure ventilation: a intraoperative study in humans. J Am Coll Cardiol 27:155-160

39. Engelmann L (2004) Rechtsherzfunktion bei ARDS und maschineller Beatmung. Internist 45:1147-1154

40. Rossi A, Polese G, Brandi G, Conti G (1995) Intrincis positive end-expiratory pressure $\left(\mathrm{PEEP}_{\mathrm{i}}\right)$. Intensive Care Med 21:522-536

41. Vieillard-Baron A, Jardin F (2003) The issue of dynamic hyperinflation in acute respiratory distress syndrome patients. Eur Respir J 22(Suppl 42):43S-47S

42. De Durante G, del Turco M, Rustichina L et al (2002) ARDSNet lower tidal volume ventilatory strategy may generate intrinsic positive end-expiratory pressure in patients with acute respiratory distrss syndrome. Am J Respir Crit Care Med 165:12711274

43. Mercat A, Diehl JL, Michard F et al (2001) Extending inspiratory time in acute respiratory distress syndrome. Crit Care Med 29:40-44

44. Tharratt RS, Allen RB, Albertson TF (1988) Pressure controlled inverse ratio ventilation in severe adult respiratory failure. Chest 94:755-762

45. Laffey JG, O'Croinin D, McLoughlin P, Kavanagh BP (2004) Permissive hypercapnia - role in protective lung ventilatory strategies. Intensive Care Med 30:347-356

46. Carvalho CRR, Barbas CSV, Medeiros DM et al (1997) Temporal hemodynamic effects of permissive hypercapnia associated with ideal PEEP in ARDS. Am J Respir Crit Care Med 156:1458-1466

47. Pfeiffer B, Hachenberg T, Wendt M, Marshall B (2002) Mechanical ventilation with permissive hypercapnia increases intrapulmonary shunt in septic and nonseptic patients with acute respiratory distress syndrome. Crit Care med 30:285-289 
48. Weber T, Tschernich H, Sitzwohl C et al (2000) Tromethamine puffer modifies the depressent effect of permissive hypercapnia on myocardial contractility in patients with acute rspiratory distress syndrome. Am J Respir Crit Care Med 162:1361-1365

49. Böhm S, Lachmann B (1996) Pressure-control ventilation. Putting a mode into perspective. Int J Intensive Care 3:12-27

50. Rappaport SH, Shpiner R, Yoshihara $\mathrm{G}$ et al (1994) Randomized, prospective trial of pressure-limited vs volume-controlled ventilation in severe respiratory failure. Crit Care Med 22: 22-32

51. Esteban A, Alia I, Gordo F et al (2000) Prospective randomized trial comparing pressure-controlled ventilation and volume-controlled vemtilation in ARDS. Chest 117:1690-1696

52. Bein T, Prasser C, Philipp A, Muller T, Weber F, Schlitt HJ, Schmid FX, Taeger K, Birnbaum D (2004) Pumpenfreie extrakorporale Lungenunterstützung mit arteriovenösem Shunt beim schweren akuten Lungenversagen des Erwachsenen. Anaesthesist 53:813-819

53. Krishnan JA, Brower RG (2000) High-frequency ventilation for acute lung injury and ARDS. Chest 118: 795-807

54. Muders F, Pfeifer M, Müller T (2003) Methodik und Anwendung der Hochfrequenzoszillationsventilation (HFOV) bei akuter Lungenschädigung und ARDS. Intensivmed 40: 515-522

55. Derdak S, Mehta S, Stewart TE et al (2002) High-frequency oscillatory ventilation for acute respiratory distress syndrome in adults. A randomized, controlled trial. Am J Respir Crit Care Med 166:801-808

56. Pelosi $\mathrm{P}$, Cadringher $\mathrm{P}$, Bottino $\mathrm{N}$ et al (1999) Sigh in acute respiratory distress syndrome. Am J Respir Crit Care Med 159:872-880

57. Putensen $\mathrm{C}$, Zech $\mathrm{S}$, Wrigge $\mathrm{H}$ et al (2001) Long-term effect of spontaneous breathing during ventilatory support in patients with acute lung injury. Am J Respir Crit Care Med 164:43-49

58. Foti G, Cereda M, Sparacino ME et al (2000) Effects of periodic lung recruitment meneuvers on gas exchange and respiratory mechanics in mechanically ventilated acute respiratory distress syndrome (ARDS) patients. Intensive Care Med 26:501-507

59. Lachmann B (1992) Open up the lung and keep the lung open. Intensive Care Med 18:319-321
60. Knothe C, Huber T, Hiltl $\mathrm{P}$ et al (2000) Beatmung nach dem „openlung"-Konzept bei polytraumatisierten Patienten. Anästhesiol Intensivmed Notfallmed Schmerzther 35:306315

61. Schreiter D, Reske A, Stichert B et al (2004) Alveolar recruitment in combination with sufficient positive endexpiratory pressure increases oxygenation and lung aeration in patients with severe chest trauma. Crit Care med 32:968-975

62. Engelmann L (2000) Das Open-lungKonzept. Anaesthesist 49:1046-1053

63. Wrigge $\mathrm{H}$, Zinserling J, Stüber $\mathrm{F}$ et al (2000) Effects of mechanical ventilation on release of cytokines into systemic circulation in patients with normal pulmonary function. Anesthesioly 93:1413-1417

64. Pötz FB, Vreugdenhil HAE, Slutsky AS et al (2002) Mechanical ventilation alters the immune response in children without lung pathology. Intensive Care Med 28:486-492

65. Tremblay L, Vaalenza F, Ribeiro SP, Li J, Slutsky AS (1997) Injurious ventilatory strategies increase cytokines and c-fos m-RNA expression in an isolated rat lung model. J Clin Invest 99:944-952

66. Held HD, Boettcher S, Hamann L, Uhlig S (2001) Ventilation-induced chemokine and cytokine release is associated with activation of nuclear factor $-\kappa \mathrm{B}$ and is blocked by steroids. Am J Respir Crit Care Med 163:711716

67. Zapol WM, Snider MT, Hill JD et al (1979) Extracorporeal membrane oxygenation in severe acute respiratory failure. A randomised prospective study. JAMA 242:2193-2196

68. Morris AH, Wallace CJ, Menlove RL et al (1994) Randomized clinical trial of pressure-controlled inverse ratio ventilation and extracorporeal $\mathrm{CO}_{2}$ removal for adult respiratory dirstress syndrome. Am J Respir Crit Care Med 149:295-305

69. Lewandowski K (2000) Extracorporeal membrane oxygenation for severe acute respiratory failure. Crit Care 4:156-168

70. Anzueto A, Baughman RP, Guntupalli KK et al (1996) Aerosolized surfactant in adults with sepsis-induced acute respiratory distress syndrome. N Engl J Med 334:1417-1421

71. Gregory TJ, Steinberg KP, Spragg R et al (1997) Bovine surfactant therapy for patients with acute respiratory distress syndrome. Am J Respir Crit Care Med 155:1309-1315
72. Spragg RG (2004) Current status of surfactant treatment of ARDS/ALI. Appl Cardiopulm Pathophysiol 13:88

73. Walmrath D, Grimminger F, Pappert D et al (2002) Bronchoscopic administration of bovine natural surfactant in ARDS and septic shiock: impact on gas exchange and haemodynamics. Eur Respir J 19:805-810

74. Deres FL, Rommelheim K (1997) Surfactant und hochdosiertes Ambroxol nach Aspiration bei Sectioeinleitung. Anästhesiol Intensivmed 9:454-461

75. Hoheisel G, Moche M, Borte G et al (1997) Surfactantgabe und seitengetrennte Überdruckbeatmung bei akutem Lungenversagen und Atelektase nach septischem Abort. Pneumologie 51:270-273

76. Abraham E, Park YC, Covington P et al (1996) Liposomal prostaglandin E1 in acute respiratory distress syndrome: a placebo-controlled, randomized, double-blind, multicenter clinical trail. Crit Care Med 24:10-15

77. Vincent JL, Bras R, Santman $F$ et al (2001) A multi-centre, double-blind, placebo-controlled study of liposomal prostaglandin E1 (TLC C-53) in patients with acute respiratory distress syndrome. Intensive Care Med 27: 1578-1583

78. Walmrath D, Olschewski H, Grimminger F, Seeger W (1997) Vasodilatative Prostanoide von der Infusion zum Aerosol: neue Perspektiven für das ARDS und die primäre pulmonale Hypertonie. Intensivmed 34:370380

79. Lundin S, Mang H, Smithies $M$ et al (1999) Inhalation of nitric oxide in acute lung injury: results of a European multicentre study. Intensive Care Med 25:911-919

80. Taylor RW, Zimmerman JL, Dellinger RP et al (2004) Low-dose inhaled nitric oxide in patients with acute lung injury. JAMA 291:1603-1609

81. Germann P, Braschi A, Della Rocca G et al (2005) Inhaled nitric oxide therapy in adults: European expert recommendations. Intensive Care Med 31:1029-1041

82. Ely EW, Baker AM, Dunagan DP et al (1996) Effect on the duration of mechanical ventilation of indentifying patients capable of breathing spontaneously. N Engl J Med 335:1864-1869

83. Engelmann L, Petros S, Gundelach K, Wegscheider K (2006) Die Bedeutung des "second hit" für den Sepsisverlauf. Intensivmed (im Druck)

84. Collective task force (2001) Evidence - based guidelines for weaning and discontinuing ventilatory support. Chest 120:375S-395S 